\title{
Deterioro cognitivo y
}

recuperación espontánea en pacientes con

diagnóstico de Consumo

Perjúdicial o síndrome de Dependencia Alcohóliso

Cognitive impairment and spontaneous recovery in patients with diagnosis

of alcohol harmful use or alcohol dependence syndrome

(c) (i) (2) (2)

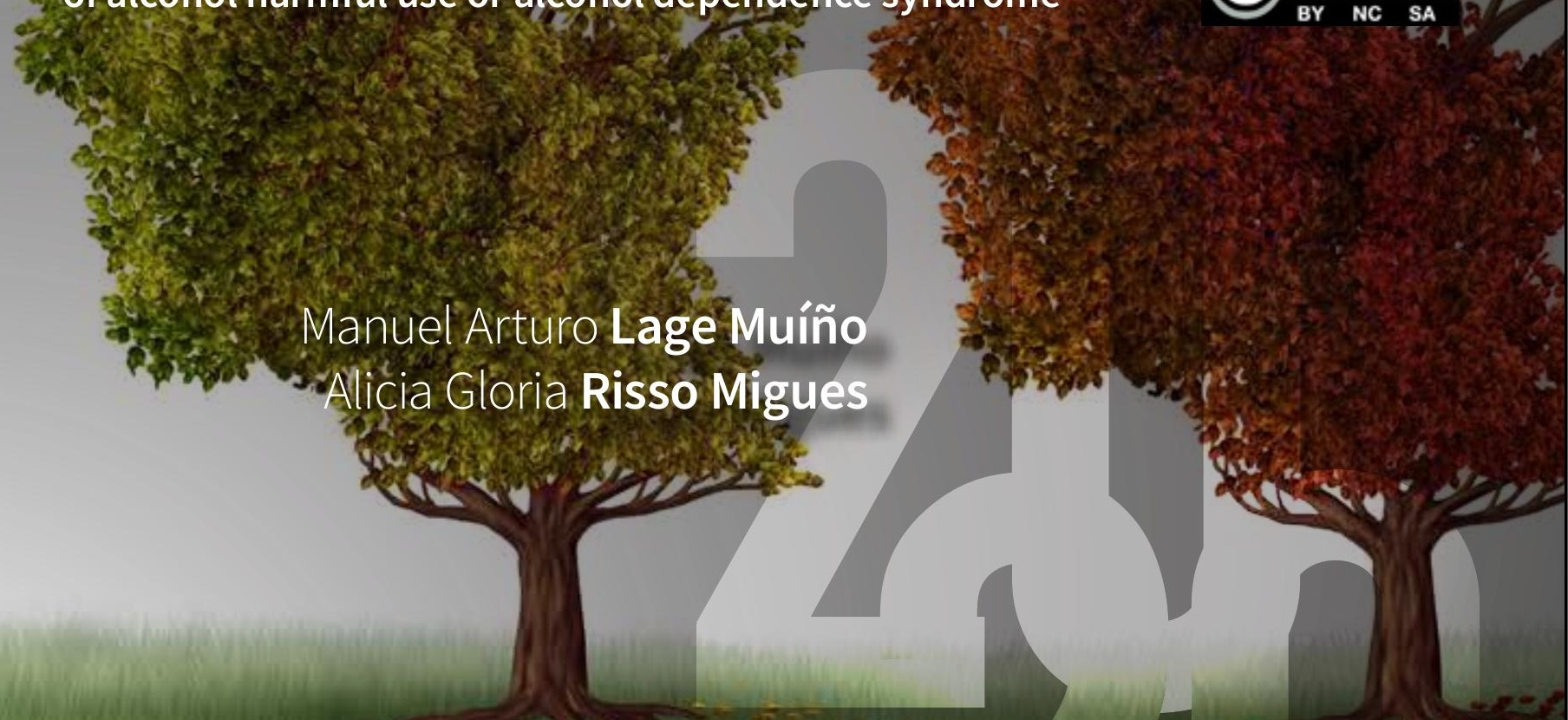

Revista Iberoamericana de

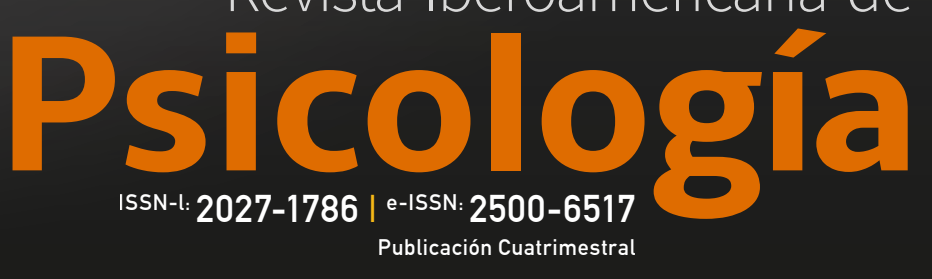


Revista Iberoamericana de

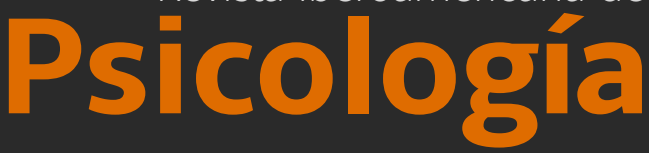

ISSN-I: 2027-1786 | e-ISSN: 2500-6517 Publicación Cuatrimestral

ID:

10.33881/2027-1786.rip.13315

Title:

Cognitive impairment and spontaneous recovery in patients with diagnosis of alcohol harmful use or alcohol dependence syndrome

Título: Deterioro cognitivo y recuperación espontánea en pacientes con diagnóstico de Consumo Perjudicial o Síndrome de Dependencia Alcohólico

Alt Title / Título alternativo:

[en]

Cognitive impairment and spontaneous recovery in patients with diagnosis of alcohol harmful use or alcohol dependence syndrome

[es]

Deterioro cognitivo y recuperación espontánea en pacientes con diagnóstico de Consumo Perjudicial o Síndrome de Dependencia Alcohólico

Author (s) / Autor (es):

Lage Muíño \& Risso Migues

Keywords / Palabras Clave:

[en]

alcohol use disorder; cognitive impairment; spontaneous remission; cognitive rehabilitation; detoxification; cognitive assessment

[es]: alcoholismo; deterioro cognitivo; remisión espontánea; rehabilitación cognitiva; desintoxicación; evaluación cognitiva

Proyecto / Project:

Tesis doctoral desarrollada dentro del Progrma de Doctorado en Desarrollo Psicológico, Aprendizaje y Salud. Escuela Internacional de Doctorado Universidad de A Coruña (España)

Submited: 2020-05-18

Acepted: 2020-08-24

\section{Resumen}

El objetivo de este trabajo era conocer el alcance del deterioro de las funciones cognitivas debido al consumo abusivo de alcohol y su posible recuperación espontánea, así como observar la evolución temporal de esta recuperación, desde el inicio hasta los 24 meses de la abstinencia. Participaron voluntariamente 100 pacientes de ambos sexos, en tratamiento de deshabituación

de consumo perjudicial o síndrome de dependencia alcohólico, agrupados en cohortes según tiempo de abstinencia. El grupo control estuvo formado por 20 personas sin antecedentes de alcoholismo, equiparadas

con los pacientes en las variables edad,

sexo y nivel de estudios. Los datos sociodemográficos y clínicos se recogieron con una anamnesis protocolizada, mientras que para evaluar el deterioro cognitivo se emplearon

el Mini-Mental State Examination (MMSE) y el

Montreal Cognitive Assessment (MoCA), en sus versiones al español. Para cada participante se obtuvieron datos de 8 variables clínicas y socio-demográficas y 27 variables cognitivas.

Se observaron diferencias significativas

entre las medidas de las mismas variables

cognitivas proporcionadas por ambos

instrumentos, siendo más discriminativo

el MoCA. Los resultados mostraron que el deterioro y la recuperación espontánea varían para las distintas funciones cognitivas y que los cambios más significativos se dan seis meses después de iniciada la deshabituación y entre los 18 y 24 meses. En base a la

evidencia obtenida, atención, memoria y lenguaje se perfilan como las funciones más susceptibles de beneficiarse de un programa de rehabilitación cognitiva, que convendría implementar entre los 6 y 18 meses de abstinencia.

\section{Abstract}

The aim of this study was to determine the extent of the deterioration of cognitive

functions due to alcohol abuse and its possible spontaneous recovery, as well as

to observe the temporal evolution of this recovery, from the beginning to 24 months

of abstinence. One hundred patients of both sexes participate voluntarily. All were in

treatment for harmful drinking cessation or alcohol dependency syndrome, and they were grouped into cohorts according to withdrawal

time. The control group consisted of 20 people with no history of alcoholism, paired with the patients in the variables of age, sex and educational level. Socio-demographic and clinical data were collected through a standardized anamnesis, while the Mini-Mental

State Examination (MMSE) and the Montreal Cognitive Assessment (MoCA), in their Spanish

versions, were used to assess cognitive

impairment. For each participant, data was obtained on 8 clinical and socio-demographic variables and 27 cognitive variables. Significant differences were observed between measures

of the same cognitive variables provided by

both instruments, with MoCA being more

discriminating. The results showed that

both the deterioration at the beginning of abstinence and its subsequent spontaneous recovery are different for each variable, and that the most outstanding changes occur 6 months after starting abstinence, and later on, between 18 and 24 months. According the obtained evidence, Attention and Language are the variables most likely to benefit from a cognitive rehabilitation program, which should be implemented between 6 and 18 months of abstinence.

\section{Citar como:}

Lage Muíño, M. A. \& Risso Migues, A. G. (2020). Deterioro cognitivo y recuperación espontánea en pacientes con diagnóstico de Consumo Perjudicial o Síndrome de Dependencia Alcohólico. Revista Iberoamericana de Psicología , 13 (3), 149-158. Obtenido de: https://reviberopsicologia.ibero.edu. co/article/view/1929

Manuel Arturo Lage Muíño, Lic

ORCID: $\quad$ https://orcid.org/0000-0003-40968609

Source I Filiacion:

Unidad de Tratamiento de Alcohol y otras Conductas Adictivas (UTACA) A Coruña (España)

BIO:

Psicólogo Clínico de la Asociación Unidad Asistencial del Alcoholismo y otras Conductas Adictivas (UTACA) de A Coruña (España) y Psicólogo privado en ejercicio.

City I Ciudad

Coruña [es]
DraAlicia Gloria Risso Migues, malic.

AutorlD: $\quad 57044484000$

Research ID: M-9798-2015

ORCID: $\quad$ https://orcid.org/0000-0001-6955-

363X

Source I Filiacion:

Universidad de A Coruña (España)

BIO:

Profesora Titular de Universidad del

Área de Metodología de las Ciencias del Comportamiento, Departamento de Psicología Universidad de A Coruña (España)

City I Ciudad:

Coruña [es] 


\section{Deterioro cognitivo y recuperación espontánea en pacientes con diagnóstico de Consumo Perjudicial o Síndrome de Dependencia Alcohólico}

Cognitive impairment and spontaneous recovery in patients with diagnosis of alcohol harmful use or alcohol dependence syndrome Manuel Arturo Lage Muíño Alicia Gloria Risso Migues

El trastorno porabuso de alcohol sueletenercomo resultado alteraciones estructurales y funcionales del sistema nervioso central y, aunque la presencia y gravedad de los déficits cognitivos tiene una alta variabilidad, se estima que entre el 50 \% y el $80 \%$ de las personas con trastornos por abuso de alcohol experimentan un deterioro cognitivo que puede ir desde leve a severo (Bates, Bowden y Barry, 2002), siendo las funciones perceptivo-motoras, viso-espaciales, de memoria y ejecutivas las que muestran mayores dificultades y más habitualmente. Algunas revisiones en torno a este tema (véase, por ejemplo, Fernández-Serrano, Pérez-García y Verdejo-García, 2011) encontraron evidencias que apuntan tanto a alteraciones neuropsicológicas (memoria episódica, procesamiento emocional y componentes ejecutivos de la toma de decisiones), como a alteraciones que se manifiestan en comportamiento impulsivo e inflexibilidad cognitiva, así como en el procesamiento espacial, velocidad perceptiva y atención selectiva. La naturaleza deestas alteraciones está en discusión, pero Moselhy, Georgiou y Kahn (2001), en un trabajo de revisión de 160 estudios muestran que el consumo abusivo de alcohol produce alteraciones en los lóbulos frontales, y hay estudios que encontraron que es en los lóbulos frontales, el hipocampo, el tálamo y el cerebelo donde el consumo abusivo de alcohol produce la mayor reducción de volumen cerebral (por ejemplo, Glass et al., 2009; Pfefferbaum, Sullivan, Mathalon y Lim, 1997; Sullivan, 2000; Yeh, Gazdzinski, Durazzo, Sjöstrand y Meyerhoff, 2007). 
En cuanto al deterioro de la memoria y las funciones ejecutivas cuando existe alcoholismo, en una revisión de los principales estudios (Landa, Fernández-Montalvo y Tirapu Ustarroz, 2004) se sugiere la existencia de una alteración de la capacidad mnésica, que afecta fundamentalmente o a la memoria de trabajo y a las estrategias de aprendizaje. Así mismo, los resultados muestran un déficit de las funciones ejecutivas en personas con abuso o dependencia del alcohol, planteándose la posibilidad de que sea un factor de vulnerabilidad a la hora de desarrollar una dependencia alcohólica (Rojo-Mota, Pedrero-Pérez, Ruiz-Sánchez de León, Llanero-Luque y Puerta-García, 2013). Particularmente preocupante, por la vulnerabilidad debida a la inmadurez del cerebro, es el caso de los adolescentes, ya que se estima que el 40 $\%$ de quienes comienzan a beber antes de los 15 años desarrollarán problemas relacionados con el consumo de alcohol (Golpe, Isorna, Barreiro, Brana y Rial, 2017; Vargas-Martinez et al., 2018; Zeigler et al., 2005). Especialmente relevantes, y conocidos desde hace tiempo, son los cambios que ocurren a consecuencia de ello en zonas como el hipocampo o el neocórtex, ya que están implicadas en procesos atencionales, mnémicos o ejecutivos (Beracochea, Durkin y Jaffard, 1986; Victor, 1994), necesarios para un adecuado desarrollo evolutivo y que, si ven comprometidos por el abuso de alcohol, podrían desembocar en un trastorno neurocognitivo caracterizado por déficits que persistirían una vez superada la abstinencia aguda (Gongvatana et al., 2014).

Por otra parte, múltiples estudios apuntan que quienes inician un tratamiento para su adicción presentan lo que Goldman describió como "niebla cognitiva" (Goldman, 1990). De hecho, hay trabajos que indican que el daño cerebral producido por la neurotoxicidad es más intenso a nivel de hipocampo, hipotálamo y cerebelo, por lo que afecta tanto a la memoria como a la capacidad de aprendizaje (Ridley, Draper y Withall, 2013), y que los daños relacionados con el déficit de tiamina, como los relacionados con la memoria anterógrada, serían más persistentes (Sachdeva, Chandra, Choudhary, Dayal y Anand, 2016).

Además, investigaciones sobre los elementos que dificultan o impiden la rehabilitación de adictos han mostrado el elevado poder predictivo de los déficits cognitivos, relacionándolo con implicaciones clínicas de gran importancia. Concretamente, se ha observado que el deterioro cognitivo en adictos en tratamiento está asociado a una baja adhesión al tratamiento (Bates, Pawlak, Tonigan y Buckman, 2006), menos probabilidades de completarlo (Fals-Stewart y Schafer, 1992), menor compromiso con el mismo (Katz et al, 2005), mal cumplimiento de las normas y pautas (Fals-Stewart, 1993), incremento de la probabilidad de una pronta recaída (Bowden-Jones, McPhillis, Rogers, Hutton y Joyce, 2005; Passetti, Clark, Mehta, Joyce y Kinga, 2008), pobre adquisición de habilidades para rehusar el consumo (Smith y McCrady, 1991), peor disposición para el cambio (Blume, Schmaling, y Marlatt, 2005), peor cumplimiento de pautas (Streeter et al. 2008), menor atribución de autoeficacia (Bates et al., 2006), menor capacidad de insight (Horner, Harvey y Denier, 1999), menos capacidad para reconocer la gravedad de la adicción y los problemas asociados (Rinn, Desai, Rosenblatt y Gastfriend, 2002), menor nivel de retención (Aharonovich et al., 2006; Aharonovich, Nunes y Hasin, 2003), y peores resultados a la hora de mantener la abstinencia después del tratamiento (Aharonovich et al., 2006).

No obstante, investigaciones previas indican que las funciones cognitivas que son recuperables (que no hayan sufrido un deterioro irreversible), se recuperan en torno a los 18 meses desde que se inicia la abstinencia (Parsons, 1994; Wilson, 2002). Teniendo en cuenta todo lo dicho, la evaluación neuropsicológica de los pacientes con trastorno por abuso de alcohol permite detectar factores de riesgo que ayudan a identificar a quienes requieran programas específicos de rehabilitación neurocognitiva (Bates, Labouvie y Voelbel, 2002). Por ello, es sumamente conveniente evaluar las funciones cognitivas a todas las personas que presentan un trastorno por abuso de alcohol y que quieran iniciar un tratamiento de deshabituación (Flórez, Espandian, Villa y Sáiz, 2019; Hayes, Demirkol, Ridley, Withall y Draper, 2016).

Es evidente que la adicción al alcohol tiene que ver con una alteración del funcionamiento de un buen número de circuitos cerebrales que se manifiesta, fundamentalmente, como déficits en la memoria, la atención y las funciones ejecutivas (Volkow, Wang, Fowler y Tomasi, 2012). Estas alteraciones obstaculizan el aprovechamiento de los tratamientos, dificultando la adquisición y puesta en práctica de nuevos contenidos y destrezas. La diferencia entre el éxito o el fracaso terapéutico puede depender, entre otras cosas, de que el paciente sea consciente de sus propios déficits, capaz de razonar y entender instrucciones complejas, de planificar sus actividades diarias tomando decisiones de manera lógica y correcta, inhibiendo las respuestas impulsivas (Verdejo, Orozco, Meersmans, Aguilar y Pérez, 2004). Como consecuencia de la existencia de déficits cognitivos, los pacientes abandonan el tratamiento en fases tempranas y son incapaces de beneficiarse de tratamientos como la terapia de prevención de recaídas, que tienen fuertes demandas cognitivas (Rojo-Mota et al., 2013). Para intentar paliar este problema en la clínica, se han propuesto protocolos de evaluación de las personas que inician tratamiento (Ruiz-Sánchez de León, Pedrero-Pérez, Rojo-Mota, Llanero-Luque y Puerta-García, 2011) y programas de rehabilitación cognitiva específicamente dirigidos a esos déficits (Pedrero-Pérez et al., 2011).

En la presente investigación se decidió estudiar la recuperación cognitiva tras la abstinencia, en pacientes a tratamiento por abuso de alcohol, en el período que abarca desde los 0 meses (consumo activo) hasta los 24 meses de abstinencia (en vez de los 12 habituales) por dos motivos. En primer lugar, porque los déficits cognitivos relacionados con el alcohol podrían influir en el aprovechamiento de los programas de tratamiento: se quería saber en qué período de la abstinencia y en qué medida se producía la recuperación, para así poder adaptar los programas a los momentos en los que dicha recuperación permitiese un mejor aprovechamiento, dentro del habitual período de intervención, que abarca de los 0 a los 24 meses. Por otro lado, también existe evidencia de la velocidad de recuperación es lenta, pero que al cabo de dos años de abstinencia tanto la mejoría como el deterioro se estabilizan, debido a que las alteraciones en la sustancia blanca son parcialmente reversibles solamente si se alcanza una abstinencia prolongada, después de la cual es cuando producen una mejoría de las funciones cognitivas y motoras (Ridley et al., 2013). Por otra parte, se quería conocer si en esta población atendida se confirma que las principales recuperaciones se producen entre los primeros meses y el primer año de abstinencia (Alhassoon et al., 2012; Bernardin, Maheut-Bosser y Paille, 2014; Erickson y White, 2009; Oscar-Berman et al., 2014; Pfefferbaum, Adalsteinsson y Sullivan, 2006), y si, como concluyeron Stavro, Pelletier y Potvin (2013) con un meta-análisis de 62 estudios, la disfunción cognitiva disminuye al cabo del año de abstinencia, o si la recuperación cognitiva no finaliza al terminar el primer año, sino que continúa más allá, llegando a producirse una recuperación significativa de las funciones deterioradas durante el segundo año de abstinencia, que era la hipótesis de partida del presente trabajo.

En base a lo anterior, el objetivo del presente trabajo es el de detectar las alteraciones de las funciones cognitivas, en personas con diagnóstico de trastorno por abuso o dependencia del alcohol que se encuentran en situación de desintoxicación o abstinencia-deshabituación, y, en su caso, la recuperación, si la hubiera, de dichos déficits, en un lapso de 24 meses desde el inicio del abandono del consumo activo, y en comparación con población no-adicta. 


\section{Método}

\section{Participantes}

La muestra se extrajo de una población formada por 2015 personas (76 \% varones) con edades comprendidas entre los 18 y los 82 años ( $M$ = 49.02, DT $=11.50)$, de pacientes en tratamiento de deshabituación de una unidad asistencial dedicada al tratamiento del alcoholismo y otras conductas adictivas de la ciudad de A Coruña (España), la cual abarca un amplio radio geográfico de residencia y un diverso origen socio-económico y cultural. La selección se realizó de forma aleatoria a partir de subgrupos poblacionales establecidos según el tiempo de abstinencia (0, 6, 12, 18y 24 meses). Los 100 participantes eran de ambos sexos (72\% de varones) y el rango de edades iba de 22 a 79 años ( $M=$ 49.57, DT $=10.79)$, sin que se apreciaran diferencias estadísticamente significativas $(p>$.05) entre estos valores y los de la población de origen. Tampoco se encontraron diferencias significativas entre la muestra y la población en las variables edad de inicio de consumo $(M=15.00, D T=$ 4.34), años de consumo problemático $(M=18.38, D T=11.19)$, y nivel de estudios (estudios universitarios: $17.5 \%$, medios: $26.67 \%$, primarios: $16.67 \%$; primaria incompleta: $39.17 \%$ ), además de en las mencionadas edad y proporción por sexo. También participaron otras 20 personas sin antecedentes ni diagnóstico de abuso de alcohol, equiparados con los pacientes en edad, sexo y nivel de estudios, formando un grupo control equivalente. Fueron seleccionadas aleatoriamente entre los familiares de los pacientes, para que cuestiones como el nivel socioeconómico y cultural, así como el lugar y tipo de residencia no difirieran significativamente de los del grupo de estudio.

Se contactó individualmente con quienes habían sido seleccionados para requerir su colaboración en la investigación. La participación fue voluntaria y se aseguró la absoluta confidencialidad, así como el respeto a las cuestiones éticas, como se detalla más adelante.

\section{Diseño}

Para el presente estudio se utilizó un modelo de Cohortes, con una muestra de 100 pacientes agrupados según el período de abstinencia: consumo activo (0 meses de abstinencia), 6, 12, 18 y 24 meses de abstinencia. Cada cohorte estuvo formada por 20 pacientes. La selección se realizó a través de un diseño de bloques homogéneos aleatorizados, siendo el tiempo de abstinencia la variable de bloqueo. Además, otros 20 participantes sin antecedentes de consumo abusivo de alcohol constituían un grupo control equivalente, formado como se indicó más arriba. mental.

Se trata de una investigación cuantitativa, de tipo cuasi-experi-

\section{Instrumentos}

Se contó con una hoja de información al paciente y de documentos de consentimiento informado y de revocación del consentimiento.

Para la recogida de variables socio-demográficas y clínicas se utilizó una anamnesis protocolizada que incluía variables clínicas y sociodemográficas: edad, estado civil, sexo, nivel de estudios, edad de inicio del consumo y años de consumo problemático de alcohol, y diagnóstico según CIE-10, en caso de detectarse patología dual.
Para la detección del deterioro cognitivo se emplearon dos instrumentos, coincidiendo con la estrategia de "usar más de un instrumento para evaluar una sola capacidad cognitiva" con el fin de "mejorar la precisión del diagnóstico” (Ortega-Marín, 2019, pp. 34-36): el Mini-Mental State Examination (MMSE) (Folstein, Folstein y McHugh, 1975; Folstein, Folstein, McHugh y Fanjiang, 2001; Lobo, Saz y Marcos, 2002; Davey y Jamieson, 2004; Feldman et al, 2008; Grupo de trabajo de la GPC, 2010; Petersen et al., 2001; Shulman et al., 2006) y el Montreal Cognitive Assessment (MoCA) (Nasreddine et al., 2005), disponible en 55 idiomas en http://www.mocatest.org/, dándose previamente de alta como clínico o investigador.

Ambos son muy utilizados, tanto en investigación como en la práctica clínica y cuentan con probada fiabilidad y validez (que oscilan entre .77 y .94, de acuerdo con la amplia literatura existente al respecto). Cabe señalar que, si bien ambos instrumentos suelen usarse para evaluar el deterioro cognitivo asociado a las demencias, y por tanto, en pacientes de edades avanzadas (véase, por ejemplo, AguilarMejía, Cárdenas-Bustos, Luengas-Monroy y Solano-Bueno, 2011 y Delgado, Araneda y Behrens, 2019), también son muy utilizados en personas más jóvenes, por ejemplo: para evaluar el deterioro cognitivo tras un traumatismo craneoencefálico (Laxe, Capdevila y Castaño, 2014), para el ictus menor o ataque isquémico transitorio (RamirezMoreno, Bartolomé, Muñoz y Guerrero, 2018), para medir el deterioro cognitivo en pacientes con esquizofrenia (Rodríguez-Bores Ramírez, Saracco-Álvarez, Escamilla-Orozco y Fresán Orellana, 2014) y, por supuesto, para evaluar el deterioro cognitivo en pacientes diagnosticados de abuso o dependencia del alcohol (Frías-Torres et al., 2018 y Rojo-Mota et al., 2013).

En total, para la presente investigación se recogieron 27 variables cognitivas que se agruparon en 10 para los análisis: atención (puntuaciones MMSE y MoCA), memoria (MMSE y MoCA), lenguaje (MMSE y MoCA), orientación (MMSE y MoCA), comprensión (MMSE) y abstracción (MoCA).

\section{Procedimiento}

A los participantes se les presentaba una hoja informativa y los documentos de consentimiento y revocación, que además les eran explicados. Luego se llevaba a cabo la recogida de datos, de forma individual y en una única sesión de aproximadamente 40 minutos. Con el fin de controlar el error progresivo, se utilizó la técnica de contrabalanceo, de forma que a la mitad de los miembros de cada cohorte (elegidos al azar) se les aplicaron las dos pruebas en un orden y a la otra mitad en orden inverso.

Para la corrección del MMSE se empleó el Manual de usuario, en español, de Revillas (2014) y para la corrección del MoCA la traducción al español de 2006 de las Instrucciones para la administración y computación de resultados, disponible en https://www. mocatest.org/

\section{Estrategia de análisis de datos}

Para el análisis de los datos se utilizó el programa IBM SPSS v24 (licencia de campus). En primer lugar, se realizaron análisis estadísticos exploratorios y se obtuvieron medidas de tendencia central y de dispersión de todas las variables de las cuales se habían recogido datos. Se procedió entonces a comparar la muestra con la población y con el grupo control en las variables de interés para la equiparación (proporción por sexos, edad, nivel de estudios), así como las cohortes 
entre sí, en lo referido a edad de inicio de consumo y tiempo de consumo problemático. Para ello se emplearon las pruebas Z, t de Student y Chi-cuadrado.

Posteriormente, dado el rango de puntuaciones máximas posibles en las distintas variables evaluadas con el MMSE y el MoCA (que podían variar entre 1 y 10), las puntuaciones brutas fueron transformadas en puntuaciones típicas ( $M=0$ y $D T=1$ ). A partir de estas puntuaciones se obtuvieron las rectas de regresión y su pendiente, para evaluar la evolución temporal del estado cognitivo global, así como el coeficiente de determinación, y se emplearon otras pruebas de estadística inferencial, concretamente análisis de varianza multivariado (incluyendo cálculos de potencia y tamaño del efecto de los contrastes) con comparaciones post-hoc (DMS), para las comparaciones inter-grupos y entre instrumentos.

\section{Consideraciones éticas}

La investigación se llevó a cabo de conformidad con las normas del Comité de Ética de la Universidad de A Coruña (España) y respeta la Ley Orgánica 3/2018 de Protección de datos personales y GDD del Estado Español, así como las recomendaciones de la Declaración de Helsinki.

\section{Resultados}

De los resultados obtenidos, el primero a destacar es que las puntuaciones totales, tanto en el MMSE como en el Moca mejoran a medida que aumenta el período de abstinencia, mostrando que el deterioro cognitivo tiende a disminuir con el paso del tiempo: $\operatorname{FMMSE}(5,114)=3.81, p=.003$ y FMoCA $(5,114)=3.85, p=.003$ (véase Figura 1).

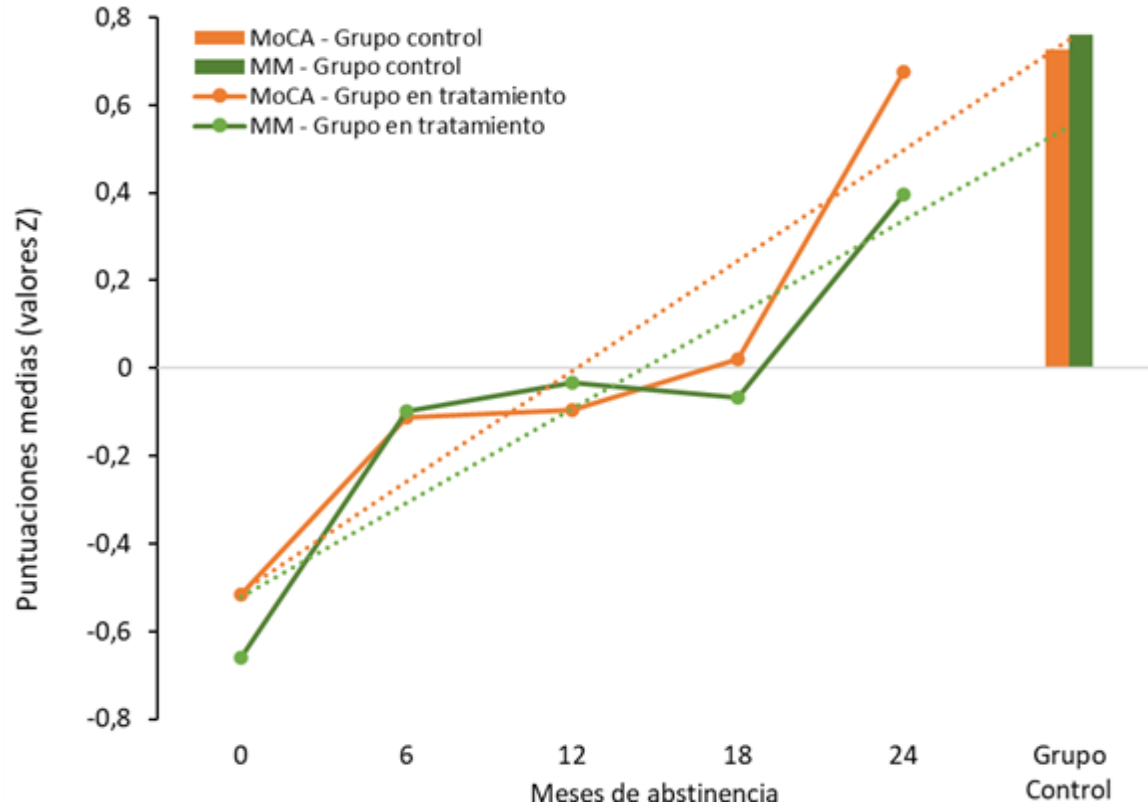

Figura 1. Evaluación del estado cognitivo global con dos instrumentos distintos (Mini Mental y MoCA), desde el inicio hasta los 24 meses de abstinencia de consumo abusivo de alcohol

No obstante, si bien en ambos contrastes la potencia estadística es muy alta (1- $\beta=$.93), el tamaño del efecto es pequeño (np2 = .24). Ello es debido a que la mejora no es constante, sino que se produce una importante recuperación desde el inicio al sexto mes de abstinencia, que después se mantiene estable hasta los 18 meses, y que vuelve mejorar de manera importante entre los 18 y 24 meses. De hecho, si se obtiene la línea de tendencia, se observa que los meses de abstinencia permiten explicar el $81 \%$ de la mejora detectada con el MMSE (R2 = $.814, p<.05)$ y el $85 \%$ de la detectada con el MoCA $(R 2=.851, p<$ .05). La pendiente del cambio es muy similar para ambos instrumentos: $B M M S E=.214$ y BMoCA $=.251$, pero el perfil de ese cambio es bastante diferente: la recuperación más importante se da entre los 0 y 6 meses para el MMSE y entre los 18 y los 24 meses para el MoCA (Figura 1). Por otra parte, transcurridos 24 meses de abstinencia, el estado cognitivo general evaluado con los dos instrumentos se acerca hasta al del grupo control al punto de no diferenciarse significativamente de él. En la Tabla 1 pueden verse los resultados de las pruebas post-hoc, en los que se observan las mencionadas diferencias.

Tabla 1. Resultados de las comparaciones post-hoc (DMS) de las puntuaciones totales en MMSE y MoCA

\begin{tabular}{|c|c|c|c|c|c|c|}
\hline \multirow{2}{*}{ Instrumento } & \multirow{2}{*}{$\begin{array}{c}\text { Meses de } \\
\text { abstinencia }\end{array}$} & \multicolumn{5}{|c|}{ Diferencias de medias (valores $\mathrm{z}$ absolutos) } \\
\hline & & 0 & 6 & 12 & 18 & 24 \\
\hline \multirow{5}{*}{ MMSE } & 6 & $.560^{\star}$ & & & & \\
\hline & 12 & $.626^{\star}$ & .066 & & & \\
\hline & 18 & $.593^{\star}$ & .033 & .033 & & \\
\hline & 24 & $1.056^{*}$ & $.496^{*}$ & $.430^{*}$ & $.463^{*}$ & \\
\hline & GC & $1.420^{*}$ & $.859^{*}$ & $.793^{*}$ & $.826^{*}$ & .364 \\
\hline \multirow{5}{*}{ MoCA } & 6 & $.402^{*}$ & & & & \\
\hline & 12 & $.419^{\star}$ & .017 & & & \\
\hline & 18 & $.537^{\star}$ & .134 & .118 & & \\
\hline & 24 & $1.191^{\star}$ & $.789^{*}$ & $.772^{\star}$ & $.655^{\star}$ & \\
\hline & GC & $1.242^{*}$ & $.839^{\star}$ & $.823^{*}$ & $.705^{*}$ & .050 \\
\hline
\end{tabular}

En muchas ocasiones, para la valoración de pacientes en rehabilitación, sólo se tienen en cuenta las puntuaciones totales. Sin embargo, un resultado importante de este estudio es que la mejora no es igual para todas las variables evaluadas (Tabla 2). Así, en el momento de ini- ciar el proceso de deshabituación (abstinencia = cero meses) presentan deterioro, en comparación con el grupo control, todas las variables estudiadas, mientras que transcurridos 24 meses de abstinencia, únicamente la memoria (MMSE) sigue puntuando significativamente por debajo del Grupo Control $(t(38)=-1.864, p<.05)$. 
Tabla 2. Puntuaciones medias de las variables evaluadas, según el período de abstinencia (puntuaciones típicas: $M=0$, DT = 1)

\begin{tabular}{|c|c|c|c|c|c|c|}
\hline \multirow{2}{*}{ Subtest } & \multicolumn{5}{|c|}{ Meses de abstinencia } & \multirow{2}{*}{$\begin{array}{l}\text { Grupo } \\
\text { Control }\end{array}$} \\
\hline & 0 & 6 & 12 & 18 & 24 & \\
\hline Orientación (MMSE) & $-0.72 a$ & $0.14 b$ & $0.02 b$ & $0.02 \mathrm{ab}$ & $0.27 b$ & $0.27 \mathrm{~b}$ \\
\hline Orientación (MoCA)) & $-0.43 a$ & $0.11 b$ & $-0.02 b$ & $-0.02 b$ & $0.11 b$ & $0.25 c$ \\
\hline Atención (MMSE) & $-0.30 a$ & $-0.01 b$ & $-0.01 b$ & $-0.06 b$ & $0.19 c$ & $0.19 c$ \\
\hline Atención (MoCA) & $-0.23 a$ & $-0.08 a$ & $-0.18 a$ & $-0.18 a$ & $0.28 \mathrm{~b}$ & $0.38 b$ \\
\hline Memoria (MMSE) & $-0.30 a$ & $-0.24 a$ & $-0.14 a$ & $0.02 \mathrm{a}$ & $0.12 \mathrm{a}$ & $0.54 b$ \\
\hline Recuerdo (MoCA) & $-0.32 a$ & $-0.14 a$ & $-0.20 a$ & $-0.08 a$ & $0.35 b$ & $0.38 \mathrm{~b}$ \\
\hline Lenguaje (MMSE) & $-0.26 a$ & $-0.26 a$ & $0.13 b$ & $0.13 b$ & $0.13 b$ & $0.13 b$ \\
\hline Lenguaje (MoCA) & $-0.48 a$ & $-0.38 a$ & $-0.09 b$ & $0.06 b$ & $0.45 c$ & $0.45 c$ \\
\hline Comprensión (MMSE) & $-0.20 a$ & $0.20 \mathrm{a}$ & $0.04 b$ & $0.04 b$ & $0.16 b$ & $0.16 b$ \\
\hline Abstracción (MoCA) & $-0.36 a$ & $0.03 \mathrm{~b}$ & $0.03 b$ & $0.03 b$ & $0.13 b$ & $0.13 b$ \\
\hline
\end{tabular}

Nota: Los valores de la misma fila que no comparten el mismo índice $(a, b, c)$ son significativamente diferentes para $p<.05$ en la prueba de dos colas de igualdad para las medias de las columnas.

Así, la Orientación mejora rápida y significativamente en los primeros seis meses después de abandonar el consumo, manteniéndose prácticamente estable a partir de ahí. Y aunque el perfil de la evolución es muy similar, la puntuación en esta variable obtenida con el MMSE se iguala con la del grupo control a los 24 meses de abstinencia, mientras que la obtenida con el MoCA se mantiene por debajo. En el caso de la Atención medida con el MMSE, la recuperación es significativa a partir de los seis meses de abstinencia, igualándose con el grupo control a los 24. Cuando se evalúa con el MoCa, sin embargo, se observa que se mantiene estable los primeros 18 meses y que no es sino hasta los 24 cuando se observa una mejora significativa, que la equipara con el grupo control. El mismo patrón se observa en el Recuerdo (MoCA). En cuanto a la Memoria (MMSE), aunque no es la función más deteriorada en inicio, no se recupera de manera significativa hasta los 24 meses, momento en que sigue sin alcanzar el nivel del grupo control. El lenguaje (MoCA) se empieza a recuperar a los 12 meses de abstinencia y también se iguala con el GC a los 24. Medido con el MMSE, en cambio, alcanza la mayor recuperación ya a los 12 meses y lo mismo ocurre con la Comprensión (MMSE). La abstracción, finalmente, se recupera prácticamente de forma completa a los seis meses, al igual que la Orientación y la Atención. La evolución comentada hasta aquí se resume gráficamente en la Figura 2.

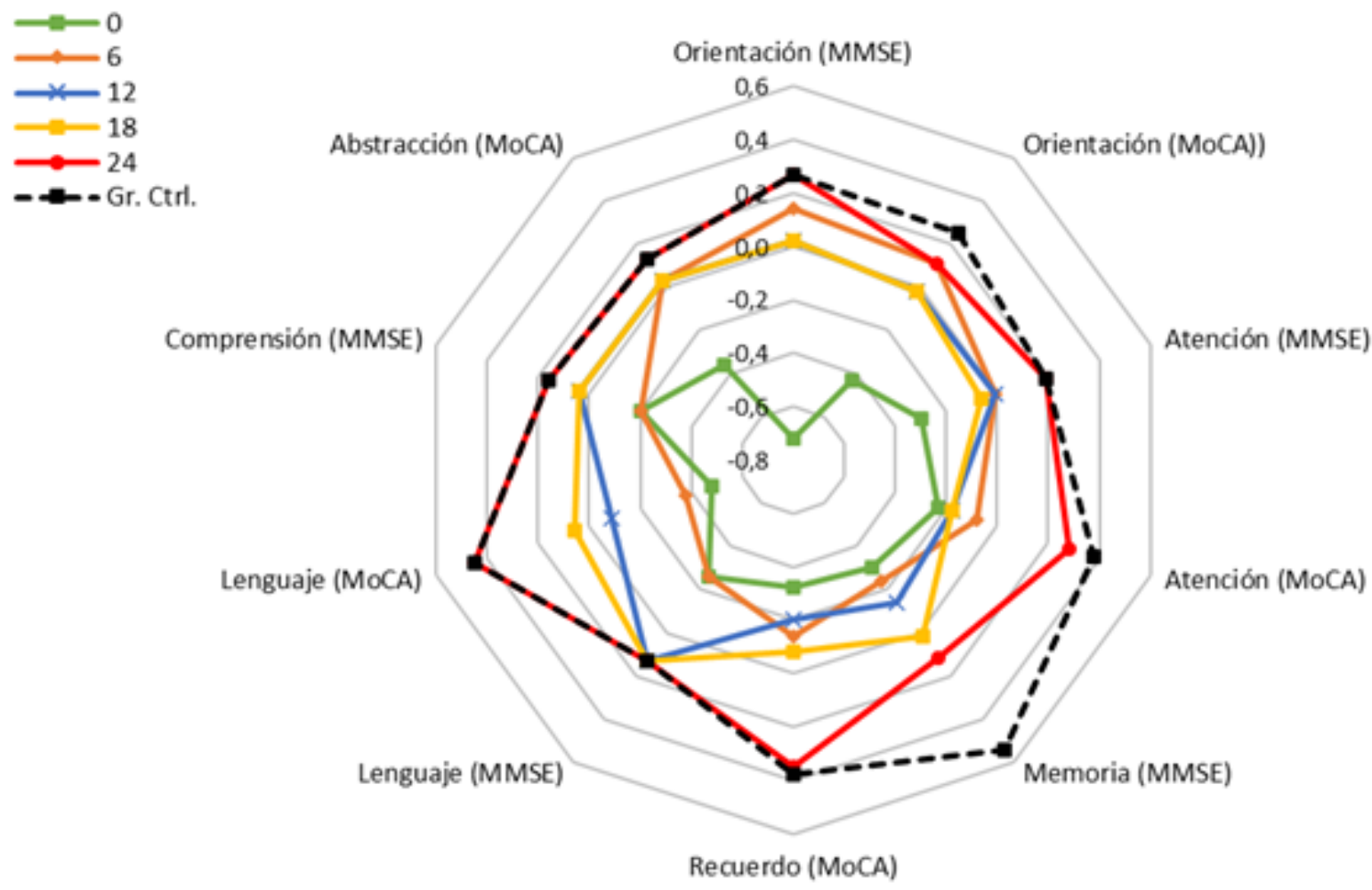

Figura 2. Evolución entre los 0 y 24 meses de abstinencia de las variables cognitivas estudiadas (en puntuaciones típicas) y comparación con el grupo control

\section{Discusión}

Con esta investigación se quería conocer el alcance del deterioro cognitivo en personas con consumo abusivo de alcohol, distinguiendo entre las distintas funciones y analizando la evolución de su posible recuperación, desde el inicio del tratamiento de desintoxicación hasta los 24 meses de abstinencia acumulada.
El deterioro cognitivo causado por el abuso de alcohol puede afectar tanto al proceso de deshabituación como a los hábitos que influyen en la salud de los pacientes, por eso es recomendable hacer una valoración de las funciones cognitivas (Flórez et al., 2019) a todas las personas que presentan un trastorno por abuso de alcohol y que quieran iniciar un tratamiento de deshabituación, concretamente tras alcanzar entre 1 y 6 semanas de abstinencia (Hayes et. al, 2016). 
En el presente trabajo, utilizando dos de los instrumentos más habituales para ello, se encontraron diferencias significativas entre las medidas de las mismas variables cognitivas proporcionadas por ambos, pareciendo ser más discriminativo el MoCA. En ese sentido, y aunque no era un objetivo prioritario de este estudio, los datos aportados confirman la hipótesis de que el test MoCA es más discriminativo que el MMSE, lo que permite decir que es un instrumento más adecuado para la evaluación del deterioro cognitivo provocado por el consumo abusivo de alcohol (coincidiendo con lo apuntado al respecto por otros autores, como Rojo-Mota et al., 2013), y que su capacidad predictiva es superior. Para ahondar más en este aspecto, en futuras investigación podrían realizarse análisis de validez convergente y discriminante.

En cuanto a los resultados relativos a cada una de las distintas variables evaluadas, estos revelan que orientación, atención, memoria, lenguaje, comprensión y abstracción se ven afectadas por el consumo abusivo de alcohol, pero también indican que mejoran significativamente con la abstinencia.

Estos hallazgos son coherentes, por una parte, con estudios que indican que las funciones cognitivas son recuperables (Wilson, 2002). Por otro lado, apoyan hallazgos mencionados anteriormente (Golpe et al., 2017; Vargas-Martínez et al., 2018), que apuntan a que el daño cerebral afecta a la memoria y a la capacidad de aprendizaje, así como los relativos al deterioro de la memoria anterógrada explicable por déficit de tiamina (Sachdeva et al., 2016). Por otra parte, el consumo prolongado de alcohol, produciría un daño de la neurotrasmisión colinérgica en el cerebro anterior basal, que juega un papel muy importante en la atención, el aprendizaje y la memoria (Ridley et al, 2013).

Así mismo, y a pesar de que el trastorno neurocognitivo puede persistir más allá de las tres semanas de abstinencia aguda (Gongvatana et al., 2014), la recuperación tras la abstinencia se produce, según nuestros resultados, de manera muy evidente de los 0 a los 6 meses de abstinencia. Después, entre los 6 y los 18 meses habría un período de un año en los que la mejoría sería mínima, para volver a producirse una clara mejoría entre los 18 y los 24 meses de abstinencia. A partir de ese lapso se estabilizaría, como se mencionó antes (Ridley et al., 2013).

Por otro lado, ya Parsons (1994) había adelantado que aquellas funciones que no han sufrido un deterioro irreversible se recuperan entorno a los 18 meses desde que se inicia la abstinencia, lo que queda evidenciado en el estudio que aquí se presenta, mostrando, también, que las investigaciones que solo llegan hasta los 18 meses no permiten observar el salto en la mejoría que se produce entre los 18 y los 24 en algunas variables.

Además, de las tres funciones cognitivas esenciales para la reincorporación socio-laboral (atención, memoria y lenguaje), la memoria no acaba de recuperarse de manera espontánea, ya que a los 24 meses de abstinencia sigue sin alcanzar la puntuación del grupo control. La atención y el lenguaje sí se recuperan pero, vista su evolución, puede postularse que lo harían más rápidamente con algún programa de rehabilitación específico, el cual sería aún más necesario en el caso de la memoria. En ese sentido, los resultados apoyan lo que afirman algunos autores sobre la conveniencia de implementar de manera sistemática, en las unidades de tratamiento de personas con problemas de abuso de alcohol, protocolos de evaluación de deterioro cognitivo (Ruiz-Sánchez de León et al., 2011), y programas de rehabilitación cognitiva, específicamente dirigidos a la recuperación de los déficits causados por el consumo (Pedrero-Pérez et al., 2011).

Quizá, la principal contribución del presente trabajo ha sido poder comprobar que la recuperación de los daños en las funciones cognitivas, deterioradas por el consumo de alcohol, va más allá de los 18 meses de abstinencia, llegando hasta, como mínimo, los dos años.
También haber obtenido apoyo sobre la importancia de evaluar por separado las distintas funciones y no de forma global, como se hace con frecuencia, ya que tanto el deterioro inicial como la mejora es significativamente diferente dependiendo de cuál se trate. Como limitación, podría señalarse la relativa a la concreción de la muestra. No obstante, dado que el estudio se centraba en variables cognitivas, no existen razones para presuponer que su deterioro y recuperación espontánea tengan que ver con cuestiones como la procedencia geográfica o el centro de tratamiento. En cualquier caso, investigaciones previas en las que se observaron resultados similares, así como las que se vayan sumando, permitirán aumentar la validez externa de las conclusiones.

Como conclusión final, podría decirse que se debe recomendar medir el deterioro cognitivo causado por el abuso de alcohol, identificándolo por funciones, y que, de entre las herramientas más utilizadas en la clínica diaria, el MoCA es el instrumento más discriminativo para evaluarlo. Además, los resultados obtenidos pueden ser de utilidad para desarrollar programas de intervención en rehabilitación cognitiva, que convendría implementar entre los 6 y 18 meses de abstinencia, siendo atención, memoria y lenguaje las que se perfilan como las funciones más susceptibles de beneficiarse de ello. Esto abre una interesante línea de investigación para futuros estudios y, en este sentido, los autores esperan poder dar a conocer, en un futuro próximo, un programa con esas características, sencillo y económico, así como los resultados de su implementación.

\section{Referencias}

Aguilar-Mejía, O., Cárdenas-Bustos, L., Luengas Monroy, F., \& Solano-Bueno, Y. (2011). Intervención neuropsicológica para adultos mayores con deterioro cognitivo leve amnésico: Estudio de Caso. Revista Iberoamericana de Psicología, 4(1), 33-40. https://reviberopsicologia. ibero.edu.co/article/view/rip.4104

Aharonovich, E., Hasin, D. S., Brooks, A. C, Liu, X, Bisaga, A., \& Nunes, E. V. (2006). Cognitive deficits predict low treatment retention in cocaine dependent patients. Drug and Alcohol Dependence, 81(3), 313-322. https://doi.org/10.1016/j.drugalcdep.2005.08.003

Aharonovich, E., Nunes, E., \& Hasin, D. (2003). Cognitive impairment, retention and abstinence among cocaine abusers in cognitivebehavioral treatment. Drug and Alcohol Dependence, 71(2), 207-211. https://doi.org/10.1016/S0376-8716(03)00092-9

Alhassoon, O. M., Sorg, S. F., Taylor, M. J., Stephan, R. A., Schweinsburg, B. C., Stricker, N. H., Gongvatan, A., \& Grant, I. (2012). Callosal white matter microstructural recovery in abstinent alcoholics: a longitudinal diffusion tensor imaging study. Alcoholism: Clinical and Experimental Research, 36(11), 1922-1931. https://doi.org/10.1111/ j.1530-0277.2012.01808.x

Bates, M. E., Bowden, S. C., \& Barry, D. (2002). Neurocognitive impairment associated with alcohol use disorders: implications for treatment. Experimental and Clinical Psychofarmalogy, 10(3), 193-212. https:// doi.org/10.1037/1064-1297.10.3.193

Bates, M. E., Labouvie, E. W., \& Voelbel, G. T. (2002). Individual differences in latent neropsychological abilities at addictions treatment entry. Psychology of Addictive Behaviors 16(1):35-46. https://doi. org/10.1037//0893-164X.16.1.35

Bates, M. E., Pawlak, A. P., Tonigan, J. S., \& Buckman, J. F. (2006). Cognitive impairment influences drinking outcome by altering therapeutic mechanisms of change. Psychology of Addictive Behaviors, 20(3), 241 253. https://doi.org/10.1037/0893-164X.20.3.241

Beracochea, D., Durkin, T. P., \& Jaffard, R. (1986). On the involvement of the central cholinergic system in memory déficits induced by longterm etanol consumption in mice. Pharmacology Biochemistry and Behavior 24(3), 519-524. https://doi.org/10.1016/0091-3057/86)90551-4 
Bernardin, F., Maheut-Bosser, A., \& Paille, F. (2014). Cognitive impairments in alcohol-dependent subjects. Frontiers in psychiatry, 5. https://doi. org/10.3389/fpsyt.2014.00078

Blume, A. W., Schmaling, K. B., \& Marlatt, G. A. (2005). Memory, executive cognitive function, and readiness to change drinking behavior. Addictive Behaviors 30(2), 301-314. https://doi.org/10.1016/j. addbeh.2004.05.019

Bowden-Jones, H., McPhillis, M., Rogers, R., Hutton, S., \& Joyce, E. (2005). Risk-Taking on tests sensitive to ventromedial prefontal cortex dysfunction predicts early relapse in alcohol dependency: a pilot study. Journal of Neuropsichiatry 17(3), 417-420. https://doi. org/10.1176/appi.neuropsych.17.3.417

Davey, R. J., \& Jamieson, S. (2004). The validity of using the mini mental state Examination in NICE dementia guidelines. Journal of Neurology, Neurosurgery \& Psychiatry 75(2), 343-344. Recuperado de: https:// jnnp.bmi.com/content/75/2/343.2.full

Delgado, C., Araneda, A., \& Behrens, M. I. (2019). Validación del instrumento Montreal Cognitive Assessment en español en adultos mayores de 60 años. Neurología, 34, 376-385. https://doi.org/10.1016/j. nrleng.2018.12.008

Erickson, C. K., \& White, W. L. (2009). The neurobiology of addiction recovery. Alcohol Treatment Quarterly, 27(3), 338- 345. https://doi. org/10.1080/07347320903014255

Fals-Stewart, W. (1993). Neurocognitive defects and their impact of substance abuse treatment. Journal of Addictions and Offender Counseling, 13(2), 46-57. https://doi.org/10.1002/j.2161-1874.1993. tb00083.x

Fals-Stewart, W., \& Schafer, J. (1992). The relationship between length of stay in drug-free therapeutic communities and neurocognitive functioning. Journal of Clinical Psychology, 48(4), 539-543. http://doi.org/10.1002/1097-4679(199207)48:4<539::AIDJCLP2270480416>3.0.CO;2-1

Feldman, H. H., Jacova, C., Robillard, A., García, A., Chow, T., Borrie, Chertkow, H. (2008). Diagnosis and treatment of dementia: 2. Diagnosis. CMAJ, 178(7), 825-836. https://doi.org/10.1503/cmaj.070798

Fernández-Serrano, M. J., Pérez-García, M., \& Verdejo-García, A. (2011). What are the specific vs. generalized effects of drugs of abuse on neuropsychological performance? Neuroscience \& Biobehavioral Reviews, 35(3), 377-406. https://doi.org/10.1016/j.neubiorev.2010.04.008

Flórez, G., Espandian, A., Villa, R., \& Sáiz, P. (2019). Deterioro cognitivo y dependencia alcohólica, implicaciones clínicas. Adicciones, 31(1), 3-7. https://doi.org/10.20882/adicciones.1284

Folstein, M. F., Folstein, S. E., \& Mchugh, P. R. (1975). "Mini-mental state”: A practical method for grading the cognitive state of patients for the clinicians. Journal of Psychiatric Research., 12(3), 189-198. https://doi. org/10.1016/0022-3956(75)90026-6

Folstein, M. F., Folstein, S. E., McHugh, P. R., \& Fanjiang, G. (2001). MMSE. Mini-Mental State Examination: User's Guide. Psychological Assessment Resources.

Frías-Torres, C., Moreno-España, J., Ortega, L., Barrio, P., Gual, A., \& Teixidor López, L. (2018). Terapia de rehabilitación cognitiva en pacientes con trastorno por consumo de alcohol y trastorno neurocognitivo. Estudio piloto. Adicciones, 30(2). https://doi.org/10.20882/ adicciones.757

Glass, J. M., Buu, A., Adams, K. M., Nigg, J. T., Puttler, L. I., Jester, J. M., \& Zucker, R. A. (2009). Effects of alcoholism severity and smoking on executive neurocognitive function. Addiction, 104(1), 38-48. https:// doi.org/10.1111/j.1360-0443.2008.02415.x

Goldman, M. S. (1990). Experience-dependent neuropsychological recovery and the treatment of chronic alcoholism. Neuropsychology Review, 1(1), 75-101. https://doi.org/10.1007/BF01108859

Golpe, S., Isorna, M., Barreiro, C., Brana, T., \& Rial, A. (2017). Binge drinking among adolescents: prevalence, risk practices and related variables. Adicciones, 29(4), 256-267. https://doi.org/10.20882/adicciones.932
Gongvatana, A., Morgan, E. E., ludicello, J. E., Letendre, S. L., Grant, I., \& Woods, S. P. (2014). A history of alcohol dependence augments HIVassociated neurocognitive deficits in persons aged 60 and older. Journal of Neurovirology, 20(5), 505-513. https://doi.org/10.1007/ s13365-014-0277-z

Grupo de Trabajo de la GPC sobre la Atención Integral a las Personas con Enfermedad de Alzheimer y otras Demencias (2010). Guía de Práctica Clínica sobre la atención integral a las personas con enfermedad de Alzheimer y otras demencias. Barcelona: Agència de Qualitat i Avaluació Sanitàries de Catalunya. http://hdl.handle.net/11351/1272

Hayes, V., Demirkol, A., Ridley, N., Withall, A., \& Draper, B. (2016). Alcoholrelated cognitive impairment: current trends and future perspectives. Neurodenerative Disease Manegement, 6(6), 509-523. https://doi. org/10.2217/nmt-2016-0030

Horner, M.D., Harvey, R.T., \& Denier, C.A. (1999). Self-report and objetive measures of cognitive defiit in patients entering substance abuse treatment. Psychiatry Research, 86(2), 155-161. https://doi. org/10.1016/s0165-1781(99)00031-1

Katz, E. C., King, S.D., Schwartz, R. P., Weintraub, E., Barksdale, W., Robinson, R., \& Brown, B. S. (2005). Cognitive ability as a factor in engagement in drug abuse treatment. The American Journal of Drug and Alcohol Abuse, 31(3), 359-369. https://doi.org/10.1081/ADA200056767

Landa, N., Fernández-Montalvo, J., \& Tirapu Ustarroz, J. (2004). Alteraciones neuropsicológicas en el alcoholismo: una revisión sobre la afectación de la memoria y las funciones ejecutivas. Adicciones, 16(1), 41-52. https://doi.org/10.20882/adicciones.417

Laxe, S., Capdevila, E., \& Castaño, B., (2014). Instrumentos de medida más frecuentemente empleados en la valoración del traumatismo craneoencefálico. Rehabilitación, 48(3), 175-181. https://doi. org/10.1016/j.rh.2013.09.003

Lobo, A., Saz, P., \& Marcos, G. (2002). MMSE: Examen Cognoscitivo MiniMental. TEA Ediciones.

Moselhy, H.F., Georgiou, G., \& Kahn, A. (2001). Frontal lobe changes in alcoholism: A review of the literature. Alcohol and alcoholism, 36 (5), 357-368. https://doi.org/10.1093/alcalc/36.5.357

Nasreddine, Z.S., Phillips, N.A., Bédirian, V., Charbonneau, S., Whitehead, V., Collin, I., \& Chertkow, H. (2005). The Montreal Cognitive Assessment, MoCA: a brief screening tool for mild cognitive impairment. Journal of the American Geriatrics Society, 53 (4), 695-699. https://doi. org/10.1111/j.1532-5415.2005.53221.x

Ortega-Marin, J. (2019). Neuropsychological Diagnostic Accuracy: Factors That Might Lead to a Wrong Diagnosis and How to Avoid Them. Revista Iberoamericana De Psicología, 12(2), 29-38. https://doi. org/10.33881/2027-1786.rip.12203

Oscar-Berman, M., Valmas, M. M., Sawyer, K. S., Ruiz, S. M., Luhar, R. B., \& Gravitz, Z. R. (2014). Profiles of impaired, spared, and recovered neuropsychological processes in alcoholism. Handbook of Clinical Neurology, 125, 183-210. https://doi.org/10.1016/B978-0-444-626196.00012-4

Parsons, O.A. (1994). Neuropsychological measures and event-related potentials in alcoholics: interrelationships, long-term reliabilities, and prediction of resumption of drinking. Journal of Clinical Psychology, 50(1), 37-46. https://doi.org/10.1002/1097-4679(199401)50:1\%3C37::aidjclp2270500105\%3E3.0.c0;2-0

Passetti, F., Clark, L., Mehta, M. A., Joyce, E, \& Kinga, M. (2008). Neuropsychological predictors of clinical outcome in opiate addiction. Drug and Alcohol Dependence, 94(1-3), 82-91. https://doi. org/10.1016/j.drugalcdep.2007.10.008

Pedrero-Pérez, E. J, Rojo-Mota, G., Ruiz-Sánchez de León, J. M., LlaneroLuque, M, \& Puerta-García, C. (2011). Rehabilitación cognitiva en el tratamiento de las adicciones. Revista de Neurología, 52(3), 163-172. https://doi.org/10.33588/rn.5203.2010513 
Petersen, R.C., Stevens, J.C., Ganguli, M., Tangalos, E.G., Cummings, J.L., \& DeKosky S.T. (2001). Practice parameter: Early detection of dementia: mild cognitive impairment (an evidence-based review). Report of the Quality Standards Subcommittee of the American Academy of Neurology. Neurology, 56(9), 1133-1142. https://doi.org/10.1212/ WNL.56.9.1133

Pfefferbaum, A., Adalsteinsson, E., \& Sullivan, E. V. (2006). Dysmorphology and microstructural degradation of the corpus callosum: Interaction of age and alcoholism. Neurobiology of Aging, 27, 994-1009. https:// doi.org/10.1016/j.neurobiolaging.2005.05.007

Pfefferbaum A., Sullivan, E. V., Mathalon, D. H., \& Lim, K. O. (1997). Frontal lobe volume loss observed with magnetic resonance imaging in older chronic alcoholics. Alcohol: Clinical and Experimental Research 21(3), pág.521-529. https://doi.org/10.1111/j.1530-0277.1997.tb03798.x

Ramírez-Moreno, J. M., Bartolomé Alberca, S., Muñoz Vega, P., \& Guerrero Barona, E. (2018). Detección del deterioro cognitivo con la Evaluación Cognitiva de Montreal en pacientes españoles con ictus minor o ataque isquémico transitorio. Neurología. https://doi.org/10.1016/j. nrl.2018.11.001

Revillas, F. (2014). Manual de usuario. Mini-examen cognoscitivo (MEC). MiniMental State Exploration (MMSE). Valencia: General ASDE.

Ridley, N. J., Draper, B., \& Withall, A. (2013). Alcohol-related dementia: an update of the evidence. Alzheimer's Research and Therapy, 5, 3. https://doi.org/10.1186/alzrt157

Rinn, W., Desai, N., Rosenblatt, H., \& Gastfriend, D. R. (2002). Addiction denial and cognitive dysfunction: a preliminary investigation. The Journal of Neuropsychiatry and Clinical Neurosciences, 14(1), 52-57. https://doi.org/10.1176/jnp.14.1.52

Rojo-Mota, G., Pedrero-Pérez, E. J., Ruiz-Sánchez de León, J. M., LlaneroLuque, M., \& Puerta-García, C. (2013). Cribado neurocognitivo en adictos a sustancias: la evaluación cognitiva de Montreal. Revista de Neurologia,56(3), pág.129-136. https://doi.org/10.33588/ rn.5603.2012628

Ruiz-Sánchez de León, J. M., Pedrero-Pérez, E. J., Rojo-Mota, G., LlaneroLuque, M., \& Puerta-García, C. (2011). Propuesta de un protocolo para la evaluación neuropsicológica de las adicciones. Revista de Neurología, 53(8), 483-493. https://doi.org/10.33588/rn.5308.2010809

Rodríguez-Bores Ramírez, L., Saracco-Álvarez, R., Escamilla-Orozco, R., \& Fresán-Orellana, A. (2014). Validez de la Escala de Evaluación Cognitiva de Montreal (MoCA) para determinar deterioro cognitivo en pacientes con esquizofrenia. Salud Mental, 37(6), 517-522. https://doi. org/10.17711/SM.0185-3325.2014.062

Sachdeva, A., Chandra, M., Choudhary, M., Dayal, P., \& Anand, K. S. (2016). Alcohol-Related Dementia and Neurocognitive Impairment: A Review Study. International Journal of High Risk Behaviors and Addiction, 5(3), e27976. https://doi.org/10.5812/ijhrba.27976
Shulman, K. I., Herrmann, N., Brodaty, H., Chiu, H., Lawlor, B., Ritchie, K., \& Scanlan, J. M. (2006). IPA survey of brief cognitive screening instruments. International Psychogeriatrics, 18(2), 281-294. https:// doi.org/10.1017/S1041610205002693

Smith, D. E., \& McCrady, B.S. (1991). Cognitive impairment among alcoholics: Impact on drink refusal skill acquisition and treatment outcome. Addictive Behaviors, 16(5), 265-274. https://doi. org/10.1016/0306-4603(91)90019-E

Stavro, K., Pelletier, J. \& Potvin, S. (2013). Widespread and sustained cognitive deficits in alcoholism: a meta-analysis. Addiction Biology, 18(2), 203-213. https://doi.org/10.1111/j.1369-1600.2011.00418.x

Streeter, C. C., Terhune, D. B., Whitfild, T. H., Gruber, S., Sarid-Segal, O., Silveri, M. M., ... Yurgelun-Todd, D.A. (2008). Performance on the Stroop predicts treatment compliance in cocaine-dependent individuals. Neuropsychopharmacology, 33(4), 827-836. https://doi. org/10.1038/sj.npp.1301465

Sullivan E. V. (2000). Human brain vulnerability to alcoholism: Evidence from neuroimaging studies. En: V. Noronha, M. Eckardt y D. Warren (eds.), Review of NIAAA's Neuroscience and Behavioral Research Portfolio (pp. 473-508). National Institute on Alcohol Abuse and Alcoholism (NIAAA) Research Monograph No. 34.

Vargas-Martinez, A. M., Trapero-Bertran, M., Gil-Garcia, E. \& Lima-Serrano, M. (2018). Impact of the Binge Drinking (BD) in Adolescence. Are we doing it right? Adicciones, 30(2), 152-154. https://doi.org/10.20882/ adicciones.1033

Verdejo, A., Orozco, C., Meersmans, M., Aguilar, F., \& Pérez, M. (2004). Impacto de la gravedad del consumo de drogas sobre distintos componentes de la función ejecutiva. Revista de Neurología, 38, 1109-1116. https://doi.org/10.33588/rn.3812.2003592

Volkow, N.D., Wang, G.J, Fowler, J.S., \& Tomasi, D. (2012). Addiction circuitry in the human brain. Annual Review of Pharmacology and Toxicology, 52, 321-336. https://doi.org/10.1146/annurev-pharmtox-010611-134625

Wilson, B. A. (2002). Towards a comprehensive model of cognitive rehabilitation. Neuropsychological Rehabilitation, 12(2), 273-281. https://doi.org/10.1080/09602010244000020

Yeh, P. H., Gazdzinski, S., Durazzo, T. C., Sjöstrand, K., \& Meyerhoff, D. J. (2007). Hierarchical linear modeling (HLM) of longitudinal brain structural and cognitive changes in alcohol-dependent individuals during sobriety. Drug and Alcohol Dependence, 91(2-3), 195-204. https://doi.org/10.1016/j.drugalcdep.2007.05.027

Zeigler, D. W., Wang, C. C., Yoast, R. A., Dickinson, B. D., McCaffree, M. A., Robinowitz, C. B., \& Sterling, M. L. (2005). The neurocognitive effects of alcohol on adolescents and college students. Preventive Medicine, 40(1), 23-32. https://doi.org/10.1016/j.ypmed.2004.04.044 